\title{
Cardiovascular diseases among adults in Afghanistan: Prevalence and associated factors from a national household survey in 2018
}

\author{
Karl Peltzer ${ }^{1}$, Supa Pengpid ${ }^{1}$
}

\section{AFFILIATION}

1 Department of Research and Development, University of Limpopo, Limpopo, South Africa

\section{CORRESPONDENCE TO}

Karl Peltzer. Department of Research and Development, University of Limpopo, Turfloop Campus, Old Admin Block, Sovenga, Limpopo, 0727,
South Africa. E-mail: kfpeltzer@gmail.com ORCID ID: https://orcid. org/0000-0002-5980-0876

\section{KEYWORDS}

cardiovascular disease, health status, health behavior, adults, Afghanistan

Received: 31 May 2021, Revised: 19 October 2021, Accepted: 20 October 2021

https://doi.org/10.18332/popmed/143216
1.19-4.73) were associated with CVD. In addition, in gender stratified analysis, among men, higher number of adult household members (lower economic status) (AOR=3.52; 95\% CI: 1.92-6.43) and inadequate fruit and vegetable intake ( $<3$ servings/day) were associated with CVD, while among women, urban residence (AOR $=0.32 ; 95 \% \mathrm{CI}$ : 0.15-0.68) and more frequent ( $\geq 3$ servings/day) fruit and vegetable consumption (AOR=0.18; 95\% CI: 0.03-0.99) were negatively associated with CVD.

CONCLUSIONS Almost one in ten adults in Afghanistan had a CVD, and several factors associated CVD were discovered, including older age (45-65 years), current tobacco use, raised total cholesterol, and inadequate fruit and vegetable consumption.

\section{INTRODUCTION}

$\overline{\text { Globally, 31\% of all deaths were attributed to cardiovascular }}$ disease (CVDs) in 2016, including 85\% due to heart attack and stroke ${ }^{1}$. Among older adults (aged $\geq 50$ years) the topranked causes of disability adjusted life years (DALYs) were ischemic heart disease and stroke in $2019^{2}$. More than $75 \%$ die from CVDs in low-resources countries ${ }^{1}$.

In the Asian region: in China 3.3\% of the men and 3.6\% of the women reported $\mathrm{CVD}^{3}$ (age: $35-74$ years), in India the prevalence of stroke was $2.0 \%$ (age: $\geq 50$ years) ${ }^{4}$, in Iran $5.3 \%$ reported coronary heart disease (CHD) (age: 20-69 years) ${ }^{5}$, in urban areas in northern Iran 9.2\% reported CHD (age: $35-$ 70 years) $^{6}$, in rural Malaysia $4.8 \%$ had CVD (age: $\geq 18$ years) ${ }^{7}$, and in a community-based study in northwestern Pakistan $4.8 \%$ had a history of stroke ${ }^{8}$. We could not find national information on CVD in Afghanistan ${ }^{9,10}$. One in five persons died from CVDs in Afghanistan in 2016 ${ }^{11}$, and ischemic heart disease and stroke were among top 10 causes of premature mortality in Afghanistan ${ }^{12}$.

Social and demographic factors associated with CVD may include increasing age and $\operatorname{sex}^{5,13,14}$, lower socioeconomic status $^{6,13-15}$, not married ${ }^{13}$, separated/divorced ${ }^{16}$, ethnicity ${ }^{17}$, and urban residence ${ }^{5}$. Behavioral variables associated with CVD may include, tobacco use $\mathrm{u}^{14,15,18}$, exposed to indoor passive smoking ${ }^{19}$, physical inactivity ${ }^{15,20}$, low or insufficient vegetable and fruit intake ${ }^{20,21}$, psychological distress $^{22}$, and opium use ${ }^{6}$. Biological factors associated with

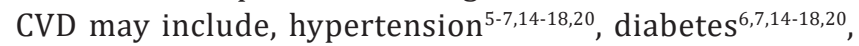
obesity $^{6,7,14,15,18,20}$, lipoprotein cholesterol, and dementia ${ }^{17}$.

This investigation aimed to estimate the prevalence and associated factors of CVDs among the national adult population in Afghanistan in 2018. 


\section{METHODS}

\section{Sample and procedure}

This analysis used data from a national cross-sectional household survey in Afghanistan in 2018 ${ }^{23}$. By using a multistage cluster approach, a nationally representative sample of individuals aged 18-69 years was generated ${ }^{24}$. The primary sampling units were 55 districts, followed by villages or blocks (secondary sampling units) and households (tertiary sampling units). One person from each household was randomly selected ${ }^{24}$. Ethical approval was obtained from the Ministry of Public Health Ethics Board in Afghanistan, and participants provided written informed consent.

Data collection followed the WHO STEPS methodology: step 1 included administration of a structured questionnaire on sociodemographic characteristics, medical history, medication use, and health risk behavior; step 2 consisted of blood pressure and anthropometric measurements; and step 3 included biochemical tests such as blood glucose and blood lipids ${ }^{24}$.

\section{Measures}

The outcome variable, having CVDs, was defined as an affirmative response to the question: 'Have you ever had a heart attack or chest pain from heart disease (angina) or a stroke (cerebrovascular accident or incident) $?^{23}$.

Sociodemographic information included age, sex, education level, number of adult household members (as a proxy for socioeconomic status) ${ }^{25}$, and residence (rural or urban).

Behavioral covariates included current tobacco use, daily servings of vegetables and fruit, sedentary behavior $(\geq 8$ hours/day) ${ }^{26}$, and physical activity (low, moderate or high) based on the Global Physical Activity Questionnaire ${ }^{27}$.

Biological variables included measured body mass index (BMI, $\mathrm{kg} / \mathrm{m}^{2}$ ) classified as: $<18.5$ underweight, 18.5-24.4 normal weight, 25-29.9 overweight, and $\geq 30$ obese $^{28}$. Hypertension was defined as systolic blood pressure $\geq 140$ $\mathrm{mmHg}$ and/or diastolic $\geq 90 \mathrm{mmHg}$ or if the participant was on antihypertensive medication ${ }^{29}$.
Diabetes was defined as: fasting plasma glucose level $\geq 7.0$ $\mathrm{mmol} / \mathrm{L}$ (126 mg/dL); or using insulin or oral hypoglycemic drugs; or having a history of diagnosis of diabetes ${ }^{30}$. Raised total cholesterol was defined as fasting total cholesterol $\geq 5.0$ $\mathrm{mmol} / \mathrm{L}$ or currently on medication for raised cholesterol ${ }^{30}$.

\section{Statistical analysis}

Descriptive statistics were used to provide the distribution of sociodemographic and health information of the sample, for both sexes and gender stratified. Unadjusted and adjusted logistic regression was applied to estimate predictors (sociodemographic and health variables) of CVDs for both sexes and multivariable logistic regression for assessing predictions of CVDs among males and females, separately. Taylor linearization methods were applied in statistical procedures accounting for sample weight and multi-stage sampling. Only complete cases were included in the analysis, and significance was set at $\mathrm{p}<0.05$. Statistical procedures were done using STATA software version 14.0 (Stata Corporation, College Station, TX, USA), and considering the complex study approach.

\section{RESULTS}

\section{Descriptive characteristics}

The sample consisted of 3956 adults (aged 18-69 years), with a median age of 35 years (interquartile range: 2460 ), and $51.9 \%$ were male. The majority (61.1\%) had no formal education, $47.4 \%$ were living with five or more adult household members, and $57.8 \%$ lived in urban areas. Two in five participants (40.3\%) were physically inactive, $44.0 \%$ engaged in sedentary behavior, $26.2 \%$ currently consumed tobacco and $59.8 \%$ had low consumption of vegetables and fruit ( $\leq 1$ servings/day). The mean BMI of respondents was $25.3 \mathrm{~kg} / \mathrm{m}^{2}, 29.2 \%$ had hypertension, $9.2 \%$ diabetes, and $18.0 \%$ raised total cholesterol. The overall proportion of CVDs was $8.8 \% ; 6.0 \%$ among females and $11.4 \%$ among males (Table 1).

\section{Table 1. The characteristics and prevalence rate of CVD in Afghanistan, STEPS survey $2018(\mathrm{~N}=3956)$}

\begin{tabular}{|c|c|c|c|c|}
\hline \multirow{3}{*}{$\begin{array}{l}\text { Characteristics (number of missing } \\
\text { data) }\end{array}$} & \multirow[t]{2}{*}{ Sample } & \multicolumn{3}{|c|}{ Cardiovascular disease (3) } \\
\hline & & All & Male & Female \\
\hline & n (\%) & n (\%) & n (\%) & n (\%) \\
\hline All & $3956(100)$ & $293(8.8)$ & $184(11.4)$ & $109(6.0)$ \\
\hline \multicolumn{5}{|l|}{ Age (years) (32) } \\
\hline $18-29$ & $1498(45.4)$ & $54(6.2)$ & $40(10.3)$ & $14(1.8)$ \\
\hline $30-44$ & $1176(32.2)$ & $95(8.6)$ & $49(9.5)$ & $46(7.7)$ \\
\hline $45-69$ & $1250(22.4)$ & $142(14.3)$ & $93(16.4)$ & $49(12.0)$ \\
\hline \multicolumn{5}{|l|}{ Education level (3) } \\
\hline None & $2225(61.1)$ & $177(9.7)$ & 85 (15.1) & $92(6.5)$ \\
\hline$\leq$ Primary & $681(15.8)$ & $48(7.1)$ & $38(7.7)$ & $10(5.7)$ \\
\hline$\geq$ Secondary & $1047(23.1)$ & $68(7.5)$ & $61(9.0)$ & $7(1.8)$ \\
\hline
\end{tabular}


Table 1. Continued

\begin{tabular}{|c|c|c|c|c|}
\hline \multirow{3}{*}{$\begin{array}{l}\text { Characteristics (number of missing } \\
\text { data) }\end{array}$} & \multirow[t]{2}{*}{ Sample } & \multicolumn{3}{|c|}{ Cardiovascular disease (3) } \\
\hline & & All & Male & Female \\
\hline & n (\%) & n (\%) & n (\%) & n (\%) \\
\hline \multicolumn{5}{|l|}{ Adult household members (3) } \\
\hline$<5$ & $2080(52.6)$ & $139(7.5)$ & $75(5.9)$ & $64(9.4)$ \\
\hline$\geq 5$ & $1873(47.4)$ & $154(9.6)$ & $109(15.2)$ & $45(3.9)$ \\
\hline \multicolumn{5}{|l|}{ Residence (1) } \\
\hline Rural & $1877(42.2)$ & $111(9.5)$ & $68(8.9)$ & $43(10.1)$ \\
\hline Urban & $2078(57.8)$ & $182(8.3)$ & $116(13.2)$ & $66(2.9)$ \\
\hline \multicolumn{5}{|l|}{$\begin{array}{l}\text { Fruit/vegetable intake (daily } \\
\text { servings) }\end{array}$} \\
\hline$\leq 1$ & $2523(59.8)$ & $192(7.9)$ & $100(8.5)$ & $92(7.4)$ \\
\hline 2 & 925 (28.9) & $78(11.4)$ & $64(15.2)$ & $14(4.4)$ \\
\hline$\geq 3$ & $508(11.3)$ & $23(6.6)$ & $20(12.0)$ & $3(1.1)$ \\
\hline \multicolumn{5}{|l|}{ Physical activity (36) } \\
\hline Low & $1489(40.3)$ & $105(6.0)$ & $50(10.1)$ & $55(4.6)$ \\
\hline Moderate & $644(17.9)$ & $44(8.1)$ & $31(7.8)$ & $13(8.6)$ \\
\hline High & $1787(41.8)$ & $141(11.8)$ & $100(13.1)$ & $41(8.3)$ \\
\hline \multicolumn{5}{|l|}{ Sedentary behavior (hours/day) (24) } \\
\hline$<8$ & $2201(56.0)$ & $155(10.4)$ & $105(12.1)$ & $49(7.9)$ \\
\hline$\geq 8$ & $1731(44.0)$ & $135(7.2)$ & $75(10.5)$ & $60(4.6)$ \\
\hline \multicolumn{5}{|l|}{ Current tobacco use (3) } \\
\hline No & $3083(73.8)$ & $201(7.0)$ & $111(9.1)$ & $90(5.7)$ \\
\hline Yes & $870(26.2)$ & $92(13.8)$ & $73(14.3)$ & $19(9.7)$ \\
\hline \multicolumn{5}{|l|}{ Hypertension (40) } \\
\hline No & $2723(70.8)$ & $157(6.9)$ & $101(9.0)$ & $56(4.4)$ \\
\hline Yes & $1193(29.2)$ & $134(13.5)$ & $81(18.0)$ & $53(9.6)$ \\
\hline \multicolumn{5}{|l|}{ Type 2 diabetes (309) } \\
\hline No & 3239 (90.8) & $214(8.5)$ & $132(11.4)$ & $82(5.2)$ \\
\hline Yes & $408(9.2)$ & $53(14.4)$ & $37(17.4)$ & $16(12.3)$ \\
\hline \multicolumn{5}{|l|}{ Raised cholesterol (248) } \\
\hline No & $3001(82.0)$ & $201(7.6)$ & $133(9.5)$ & $68(5.0)$ \\
\hline Yes & $707(18.0)$ & $72(15.4)$ & $39(26.6)$ & $33(9.3)$ \\
\hline Body mass index $\left(\mathrm{kg} / \mathrm{m}^{2}\right)$, mean $\pm \mathrm{SD}$ & $25.3 \pm 5.9$ & $26.1 \pm 5.6$ & $25.0 \pm 4.4$ & $24.9 \pm 6.3$ \\
\hline
\end{tabular}

\section{Associations with CVDs}

In adjusted logistic regression analysis showed that age 4569 years (AOR=2.06; 95\% CI: 1.06-4.01), current tobacco use (AOR=1.40; 95\% CI: 1.06-4.01) and raised total cholesterol $(A O R=2.37 ; 95 \%$ CI: 1.19-4.73) were associated with CVD (Table 2). In addition, in gender stratified analysis, among men, higher number of adult household members (lower economic status) (AOR=3.52; 95\% CI: 1.92-6.43) and inadequate fruit and vegetable intake ( $<3$ servings/day) were associated with CVD, while among women, urban residence $(\mathrm{AOR}=0.32 ; 95 \% \mathrm{CI}: 0.15-0.68)$ and more frequent $(\geq 3$ servings/day) fruit and vegetable consumption (AOR=0.18; 95\% CI: 0.03-0.99) were negatively associated with CVD (Table 3). 
Table 2. Associations with cardiovascular disease in both sexes in Afghanistan, STEPS survey 2018 (N=3626)

\begin{tabular}{|c|c|c|c|c|}
\hline Variable & OR $(95 \% \mathrm{CI})$ & $\mathbf{p}$ & AOR $(95 \% \mathrm{CI})^{\mathrm{a}}$ & $\mathbf{p}$ \\
\hline \multicolumn{5}{|l|}{ Age (years) } \\
\hline 18-29 (Ref.) & 1 & & 1 & \\
\hline $30-44$ & $1.43(0.84-2.42)$ & 0.187 & $1.27(0.74-2.16)$ & 0.385 \\
\hline $45-69$ & $2.52(1.71-3.70)$ & $<0.001$ & $2.06(1.06-4.01)$ & 0.034 \\
\hline \multicolumn{5}{|l|}{ Sex } \\
\hline Female (Ref.) & 1 & & 1 & \\
\hline Male & $2.03(0.89-4.65)$ & 0.093 & $1.69(0.66-4.31)$ & 0.272 \\
\hline \multicolumn{5}{|l|}{ Education level } \\
\hline None (Ref.) & 1 & & 1 & \\
\hline$\leq$ Primary & $0.71(0.34-1.49)$ & 0.369 & $0.56(0.27-1.22)$ & 0.145 \\
\hline$\geq$ Secondary & $0.76(0.43-1.34)$ & 0.344 & $0.62(0.33-1.15)$ & 0.130 \\
\hline \multicolumn{5}{|l|}{ Adult household members } \\
\hline$<5$ (Ref.) & 1 & & 1 & \\
\hline$\geq 5$ & $1.30(0.77-2.22)$ & 0.328 & $1.52(0.85-2.71)$ & 0.156 \\
\hline \multicolumn{5}{|l|}{ Residence } \\
\hline Rural (Ref.) & 1 & & 1 & \\
\hline Urban & $0.87(0.42-1.80)$ & 0.699 & $1.00(0.51-1.99)$ & 0.999 \\
\hline \multicolumn{5}{|c|}{$\begin{array}{l}\text { Fruit/vegetable intake (daily } \\
\text { servings) }\end{array}$} \\
\hline$\leq 1$ (Ref.) & 1 & & 1 & \\
\hline 2 & $1.50(0.89-2.52)$ & 0.127 & $1.32(0.82-2.10)$ & 0.248 \\
\hline$\geq 3$ & $0.82(0.32-2.24)$ & 0.689 & $0.80(0.31-2.01)$ & 0.626 \\
\hline \multicolumn{5}{|l|}{ Physical activity } \\
\hline Low (Ref.) & 1 & & 1 & \\
\hline Moderate & $1.39(0.61-3.17)$ & 0.436 & $1.21(0.50-2.93)$ & 0.675 \\
\hline High & $2.12(1.13-3.97)$ & 0.020 & $2.03(0.92-4.42)$ & 0.078 \\
\hline \multicolumn{5}{|c|}{ Sedentary behavior (hours/day) } \\
\hline$<8$ (Ref.) & 1 & & 1 & \\
\hline$\geq 8$ & $0.67(0.34-1.31)$ & 0.239 & $0.67(0.30-1.49)$ & 0.325 \\
\hline \multicolumn{5}{|l|}{ Current tobacco use } \\
\hline No (Ref.) & 1 & & 1 & \\
\hline Yes & $2.12(1.21-3.73)$ & 0.009 & $1.40(1.06-4.01)$ & 0.034 \\
\hline \multicolumn{5}{|l|}{ Hypertension } \\
\hline No (Ref.) & 1 & & 1 & \\
\hline Yes & $2.12(1.07-4.18)$ & 0.030 & $1.71(0.87-3.35)$ & 0.117 \\
\hline \multicolumn{5}{|l|}{ Type 2 diabetes } \\
\hline No (Ref.) & 1 & & 1 & \\
\hline Yes & $1.81(0.81-4.04)$ & 0.147 & $1.30(0.59-2.83)$ & 0.514 \\
\hline \multicolumn{5}{|l|}{ Raised cholesterol } \\
\hline No (Ref.) & 1 & & 1 & \\
\hline Yes & $2.22(1.01-4.91)$ & 0.047 & $2.37(1.19-4.73)$ & 0.014 \\
\hline Body mass index $\left(\mathrm{kg} / \mathrm{m}^{2}\right)$ & $0.99(0.95-1.04)$ & 0.746 & $0.97(0.91-1.02)$ & 0.243 \\
\hline
\end{tabular}

OR: odds ratio. AOR: adjusted odds ratio. CI: confidence interval. aVariables included those with $\mathrm{p}<0.2$ in univariate analysis. 
Table 3. Associations with cardiovascular disease among men and women in Afghanistan, STEPS survey 2018 $(\mathrm{N}=3626)$

\begin{tabular}{|c|c|c|c|c|}
\hline \multirow[t]{2}{*}{ Variable } & \multicolumn{2}{|c|}{ Male } & \multicolumn{2}{|c|}{ Female } \\
\hline & AOR (95\% CI) & $\mathbf{p}$ & AOR $(95 \% \mathrm{CI})^{\mathrm{a}}$ & $\mathbf{p}$ \\
\hline \multicolumn{5}{|l|}{ Age (years) } \\
\hline $18-29$ (Ref.) & 1 & & 1 & \\
\hline $30-44$ & $0.87(0.50-1.49)$ & 0.604 & $7.42(1.93-28.38)$ & 0.004 \\
\hline $45-69$ & $1.52(0.54-4.25)$ & 0.423 & $8.01(2.76-23.24)$ & $<0.001$ \\
\hline \multicolumn{5}{|l|}{ Education level } \\
\hline None (Ref.) & 1 & & 1 & \\
\hline$\leq$ Primary & $0.44(0.19-1.00)$ & 0.050 & $1.24(0.39-3.92)$ & 0.713 \\
\hline$\geq$ Secondary & $0.52(0.25-1.04)$ & 0.065 & $0.43(0.05-3.50)$ & 0.432 \\
\hline \multicolumn{5}{|l|}{ Adult household members } \\
\hline$<5$ (Ref.) & 1 & & 1 & \\
\hline$\geq 5$ & $3.52(1.92-6.43)$ & $<0.001$ & $0.58(0.25-1.35)$ & 0.205 \\
\hline \multicolumn{5}{|l|}{ Residence } \\
\hline Rural (Ref.) & 1 & & 1 & \\
\hline Urban & $1.69(0.73-3.53)$ & 0.223 & $0.32(0.15-0.68)$ & 0.003 \\
\hline \multicolumn{5}{|c|}{$\begin{array}{l}\text { Fruit/vegetable intake (daily } \\
\text { servings) }\end{array}$} \\
\hline$\leq 1$ (Ref.) & 1 & & 1 & \\
\hline 2 & $1.93(1.24-2.99)$ & 0.003 & $0.42(0.13-1.37)$ & 0.151 \\
\hline$\geq 3$ & $1.60(0.77-3.36)$ & 0.209 & $0.18(0.03-0.99)$ & 0.047 \\
\hline \multicolumn{5}{|l|}{ Physical activity } \\
\hline Low (Ref.) & 1 & & 1 & \\
\hline Moderate & $1.05(0.34-3.25)$ & 0.934 & $1.58(0.45-5.61)$ & 0.476 \\
\hline High & $2.18(0.86-5.52)$ & 0.101 & $1.69(0.65-4.38)$ & 0.283 \\
\hline \multicolumn{5}{|c|}{ Sedentary behavior (hours/day) } \\
\hline$<8$ (Ref.) & 1 & & 1 & \\
\hline$\geq 8$ & $0.90(0.36-2.25)$ & 0.815 & $0.71(0.39-1.26)$ & 2.40 \\
\hline \multicolumn{5}{|l|}{ Current tobacco use } \\
\hline No (Ref.) & 1 & & 1 & \\
\hline Yes & $1.24(0.50-3.10)$ & 0.637 & $0.98(0.32-3.09)$ & 0.977 \\
\hline \multicolumn{5}{|l|}{ Hypertension } \\
\hline No (Ref.) & 1 & & 1 & \\
\hline Yes & $1.76(0.93-3.35)$ & 0.082 & $1.83(0.65-5.15)$ & 0.250 \\
\hline \multicolumn{5}{|l|}{ Type 2 diabetes } \\
\hline No (Ref.) & 1 & & 1 & \\
\hline Yes & $1.20(0.41-3.69)$ & 0.737 & $1.80(0.47-6.87)$ & 0.390 \\
\hline \multicolumn{5}{|l|}{ Raised cholesterol } \\
\hline No (Ref.) & 1 & & 1 & \\
\hline Yes & $3.06(1.58-5.62)$ & $<0.001$ & $1.89(0.82-4.33)$ & 0.132 \\
\hline Body mass index $\left(\mathrm{kg} / \mathrm{m}^{2}\right)$ & $1.01(0.94-1.09)$ & 0.775 & $0.96(0.89-1.02)$ & 0.101 \\
\hline
\end{tabular}

AOR: adjusted odds ratio. CI: confidence interval. aVariables included those with $\mathrm{p}<0.2$ in univariate analysis. 


\section{DISCUSSION}

In this population-based national study among adults in Afghanistan in 2018, the prevalence of CVD (8.8\%) was higher than in China $(3.5 \%)^{3}$, Malaysia $(4.8 \%)^{7}$, Nepal $(2.0 \%)^{21}$ and Thailand $(1.6 \%)^{3}$. Public health interventions are urgently needed in Afghanistan to prevent and manage CVDs $^{31,32}$.

In line with former results ${ }^{5,13,14}$, increasing age (45-69 years) increased the odds of CVD. Unlike some previous investigations $\mathrm{s}^{5,13,14}$, this study did not find significant sex differences in the prevalence of CVD. Previous research ${ }^{5,6,13-15}$ found an association between lower socioeconomic status, and urban residence, with CVD, while this study did not show such associations, for both sexes. However, among men, lower economic status (more adult household members) was positively associated with CVD, and among women, urban residence was negatively associated with CVD.

In line with past research ${ }^{14,15,18}$, current tobacco use increased the odds of CVD. Of the stroke related risk factors, tobacco use represents the most urgent challenge for Afghanistan, since its use is high and common across gender and regions 9 . In a meta-analysis of studies, the importance of smoking as an independent risk factor for stroke was confirmed ${ }^{33}$. Consistent with previous findings ${ }^{20,21}$, we found among men and women that low or insufficient vegetable and fruit intake was associated with CVD. Contrary to expectation ${ }^{15,20}$, physical inactivity was in this study not significantly associated with CVD. It is possible that participants after having been diagnosed with CVD increased their physical activity.

Consistent with findings ${ }^{5-7,14-18,20}$, this survey showed an association between raised total cholesterol, and in univariate analysis hypertension, with CVD. High rates of total cholesterol were also found in the general population in Afghanistan suggesting high propensity for ischemic stroke in this population ${ }^{9}$. Unlike some studies ${ }^{6,7,14-18,20}$, this analysis did not find significant associations between higher BMI, and diabetes, and CVD.

\section{Strengths and limitations}

Some of the variables assessed in this study were by selfreport, including the outcome variable CVDs. However, previous research comparing hospital diagnosed and selfreported CVD, found self-reported CVD to be valid ${ }^{34}$. Another limitation was the cross-sectional design of the study, which hinders making causative conclusions. The prevalence of CVD is this survey was probably underestimated, since only survivors of CVD were included ${ }^{31}$. Furthermore, more details of the CVD type, time of CVD diagnosis and other relevant variables, such as psychological distress, were not assessed and should be part of future research.

\section{CONCLUSIONS}

Almost one in ten Afghan adults had CVD. Several associated factors for CVDs, such as older age (45-65 years), current tobacco use, raised total cholesterol, and inadequate fruit and vegetable consumption, were identified, which can be targeted in public health interventions. Local health systems need to be strengthened and mass education programs initiated.

\section{REFERENCES}

1. World Health Organization. Cardiovascular diseases (CVDs). World Health Organization. June 11, 2021. Accessed October 19, 2021. https://www.who.int/news-room/fact-sheets/ detail/cardiovascular-diseases-(cvds)

2. GBD 2019 Diseases and Injuries Collaborators. Global burden of 369 diseases and injuries in 204 countries and territories, 1990-2019: a systematic analysis for the Global Burden of Disease Study 2019. Lancet. 2020;396(10258):1204-1222. doi:10.1016/S0140-6736(20)30925-9

3. He J, Neal B, Gu D, et al. International collaborative study of cardiovascular disease in Asia: design, rationale, and preliminary results. Ethn Dis. 2004;14(2):260-268. Accessed October 19, 2021. https://www.ethndis.org/priorarchives/ ethn-14-02-260.pdf

4. Ruan Y, Guo Y, Zheng Y, et al. Cardiovascular disease (CVD) and associated risk factors among older adults in six low-and middleincome countries: results from SAGE Wave 1. BMC Public Health. 2018;18(1):778. doi:10.1186/s12889-018-5653-9

5. Abbasi M, Neishaboury M, Koohpayehzadeh J, et al. National Prevalence of Self-Reported Coronary Heart Disease and Chronic Stable Angina Pectoris: Factor Analysis of the Underlying Cardiometabolic Risk Factors in the SuRFNCD-2011. Glob Heart. 2018;13(2):73-82.e1. doi:10.1016/j.gheart.2018.01.001

6. Ghaemian A, Nabati M, Saeedi M, Kheradmand M, Moosazadeh M. Prevalence of self-reported coronary heart disease and its associated risk factors in Tabari cohort population. BMC Cardiovasc Disord. 2020;20(1):238. doi:10.1186/s12872-020-01526-w

7. Aniza I, Nurmawati A, Hanizah Y, Ahmad Taufik J. MODIFIABLE RISK FACTORS OF CARDIOVASCULAR DISEASE AMONG ADULTS IN RURAL COMMUNITY OF MALAYSIA: A CROSS SECTIONAL STUDY. Malaysian journal of public health medicine. 2016;16(1):53-61. Accessed October 19, 2021. https://www.researchgate.net/publication/305148183_ Modifiable_risk_factors_of_cardiovascular_disease_among_ adults_in_rural_community_of_Malaysia_A_cross_sectional_ study

8. Jafar TH. Blood pressure, diabetes, and increased dietary salt associated with stroke - results from a community-based study in Pakistan. J Hum Hypertens. 2006;20(1):83-85. doi:10.1038/sj.jhh.1001929

9. Bhalla D, Marin B, Preux PM. Stroke profile in Afghanistan and Nepal. Neurol Asia. 2009;14(2):87-94. Accessed October 19, 2021. https://www.neurology-asia.org/ articles/20092_087.pdf

10. Wasay M, Khatri IA, Kaul S. Stroke in South Asian countries. Nat Rev Neurol. 2014;10(3):135-143. doi:10.1038/nrneurol.2014.13 
11. World Health Organization. Noncommunicable Diseases (NCD) Country Profiles 2018. World Health Organization; 2018:28. Accessed October 19, 2021. https://apps.who.int/ iris/handle/10665/274512

12. GBD 2016 Causes of Death Collaborators. Global, regional, and national age-sex specific mortality for 264 causes of death, 1980-2016: a systematic analysis for the Global Burden of Disease Study 2016. Lancet. 2017;390(10100):1151-1210. doi:10.1016/S0140-6736(17)32152-9

13. Abdalla SM, Yu S, Galea S. Trends in Cardiovascular Disease Prevalence by Income Level in the United States. JAMA Netw Open. 2020;3(9):e2018150. doi:10.1001/jamanetworkopen.2020.18150

14.Gikas A, Lambadiari V, Sotiropoulos A, Panagiotakos D, Pappas S. Prevalence of Major Cardiovascular Risk Factors and Coronary Heart Disease in a Sample of Greek Adults: The Saronikos Study. Open Cardiovasc Med J. 2016;10:6980. doi:10.2174/1874192401610010069

15. Ribeiro ÍJS, Cardoso JP, Freire IV, Carvalho MF, Pereira R. Determinants of Stroke in Brazil: A Cross-Sectional Multivariate Approach from the National Health Survey. J Stroke Cerebrovasc Dis. 2018;27(6):1616-1623. doi:10.1016/j.jstrokecerebrovasdis.2018.01.013

16. Sanuade OA, Dodoo FN, Koram K, de-Graft Aikins A. Prevalence and correlates of stroke among older adults in Ghana: Evidence from the Study on Global AGEing and adult health (SAGE). PLoS One. 2019;14(3):e0212623. doi:10.1371/journal.pone.0212623

17. Teh WL, Abdin E, Vaingankar JA, et al. Prevalence of stroke, risk factors, disability and care needs in older adults in Singapore: results from the WiSE study. BMJ Open. 2018;8(3):e020285. doi:10.1136/bmjopen-2017-020285

18. Hennis A, Hambleton I, Fraser H, Tulloch-Reid M, Barcelo A, Hassell T. Risk factors for cardiovascular disease in the elderly in Latin America and the Caribbean. Prevention and Control. 2006;2(4):175-185. doi:10.1016/j.precon.2007.04.003

19. Shiue I. Modeling the Effects of Indoor Passive Smoking at Home, Work, or Other Households on Adult Cardiovascular and Mental Health: The Scottish Health Survey, 2008-2011. Int J Environ Res Public Health. 2014;11(3):3096-3107. doi:10.3390/ijerph110303096

20. Fuchs SC, Moreira LB, Camey SA, Moreira MB, Fuchs FD. Clustering of risk factors for cardiovascular disease among women in Southern Brazil: a population-based study. Cad Saude Publica. 2008;24(Suppl 2):S285-S293. doi:10.1590/s0102-311x2008001400013

21. Nepali S, Rijal A, Olsen MH, McLachlan CS, Kallestrup P, Neupane D. Factors affecting the fruit and vegetable intake in Nepal and its association with history of self-reported major cardiovascular events. BMC Cardiovasc Disord. 2020;20:425. doi:10.1186/s12872-020-01710-y

22. Atlantis E, Sullivan T. Changes in cardiovascular disease burden associated with psychopathology in Australian adults 2004-2008. Gen Hosp Psychiatry. 2012;34(4):345-351. doi:10.1016/j.genhosppsych.2012.02.006

23. STEPwise Approach to NCD Risk Factor Surveillance (STEPS). World Health Organization. Accessed October 19, 2021. https://www.who.int/ncds/surveillance/steps/en/

24.STEPS 2018: Afghanistan, 2018. World Health Organization, Ministry of Public Health. October 12, 2020. Updated October 12, 2020. Accessed October 19, 2021. https://extranet.who. int/ncdsmicrodata/index.php/catalog/782

25. Melki IS, Beydoun HA, Khogali M, Tamim H, Yunis KA; National Collaborative Perinatal Neonatal Network (NCPNN). Household crowding index: a correlate of socioeconomic status and inter-pregnancy spacing in an urban setting. J Epidemiol Community Health. 2004;58(6):476-480. doi:10.1136/jech.2003.012690

26. Ekelund U, Steene-Johannessen J, Brown WJ, et al. Does physical activity attenuate, or even eliminate, the detrimental association of sitting time with mortality? A harmonised meta-analysis of data from more than 1 million men and women. Lancet. 2016;388(10051):1302-1310. doi:10.1016/S0140-6736(16)30370-1

27. Armstrong T, Bull F. Development of the World Health Organization Global Physical Activity Questionnaire (GPAQ). J Public Health. 2006;14(2):66-70. doi:10.1007/s10389-006-0024-x

28. Body mass index - BMI. WHO Regional Office for Europe. Accessed September 20, 2020. https://www.euro.who.int/ en/health-topics/disease-prevention/nutrition/a-healthylifestyle/body-mass-index-bmi

29. Chobanian AV, Bakris GL, Black HR, et al. Seventh Report of the Joint National Committee on Prevention, Detection, Evaluation, and Treatment of High Blood Pressure. Hypertension. 2003;42(6):1206-1252. doi:10.1161/01.HYP.0000107251.49515.c2

30. World Health Organization. WHO STEPS Surveillance Manual: The WHO STEPwise approach to chronic disease risk factor surveillance. Updated January 26, 2017. Accessed October 10, 2020. https://www.who.int/ncds/surveillance/steps/ STEPS_Manual.pdf

31.Zaw KK, Nwe N, Hlaing SS. Prevalence of cardiovascular morbidities in Myanmar. BMC Res Notes. 2017;10(1):99. doi:10.1186/s13104-017-2422-2

32.Góngora-Rivera F. Perspective on stroke in Mexico. Medicina universitaria. 2015;17(68):184-187. doi:10.1016/j.rmu.2015.04.001

33. Peters SAE, Huxley RR, Woodward M. Smoking as a Risk Factor for Stroke in Women Compared With Men: A Systematic Review and Meta-analysis of 81 Cohorts, Including 3980359 Individuals and 42401 Strokes. Stroke. 2013;44(10):28212828. doi:10.1161/STROKEAHA.113.002342

34. Jamrozik E, Hyde Z, Alfonso H, et al. Validity of self-reported versus hospital-coded diagnosis of stroke: a cross-sectional and longitudinal study. Cerebrovasc Dis. 2014;37(4):256262. doi:10.1159/000358583 


\section{ACKNOWLEDGEMENTS}

The data source, the World Health Organization NCD Microdata Repository (URL: https://extranet.who.int/ncdsmicrodata/index.php/ catalog), is acknowledged.

CONFLICTS OF INTEREST

The authors have completed and submitted the ICMJE Form for Disclosure of Potential Conflicts of Interest and none was reported.

\section{FUNDING}

There was no source of funding for this research.

\section{ETHICAL APPROVAL AND INFORMED CONSENT}

Ethical approval for this study was not required as the data used were from an existing STEPS (2018) National Survey, for which participants provided written informed consent.

\section{DATA AVAILABILITY}

The data supporting this research are available from the following source: https://extranet.who.int/ncdsmicrodata/index.php/catalog/782

PROVENANCE AND PEER REVIEW

Not commissioned; externally peer reviewed. 